\title{
On-line compositional controller synthesis for $\mathrm{AGV}^{\star}$
}

\author{
Johan Girault • Jean-Jacques Loiseau • \\ Olivier H. Roux
}

Received: date / Accepted: date

\begin{abstract}
This paper deals with the on-line design of a supervisor to coordinate an automated guided vehicle (AGV) fleet. This supervisor ensures the system safety (no collision) and a good coordination between vehicles (no blocking situations). It is the so-called Wonham-Ramadge supervisor, it is the least restrictive, and ensures controllability and nonblocking. We propose a compositional procedure to resolve this problem allowing an efficient on-line synthesis. A calculation on the fly is made at every attribution of a new mission for an AGV, to actualize the supervisor and adapt it to the new situation. This compositional approach allows to increase the number of AGV taken on compared to the monolithic approach. We show on some tests the efficiency of this method for the on-line synthesis of supervisor to coordinate a fleet of mobile robots for real cases.
\end{abstract}

Keywords finite automata $\cdot$ control · Automated Guided Vehicle · supervisory control $\cdot$ on-line synthesis

\section{Introduction}

Automated Guided Vehicle (AGV) systems are widely spread in automatic material handling systems and production workshops. Compared to other solutions, such as the use of treadmills, the main advantage is to increase the system's flexibility and its operation requires a more complex control system.

* This paper is an extended version of Girault et al (2013) and was selected by the conference MSR'13 for this submission to the journal DEDS.

Johan Girault · Jean-Jacques Loiseau · Olivier H. Roux

Ecole Centrale de Nantes, IRCCyN, UMR CNRS 6597

1 , rue de la Noë

44321 Nantes, France

E-mail: \{johan.girault, jean-jacques.loiseau, olivier-h.roux $@$ @irccyn.ec-nantes.fr 
When designing a system for automated guided vehicles, it is required to make a good conflict-free routing of AGVs and to dispatch tasks (Reveliotis. 2000). The design of AGV fleet control systems has been the subject of many academic studies. Many solutions are based on the scheduling theory (Maza. 2003; Breton et al. 2006) where the authors merge predictive scheduling techniques with on-line reroutings to take into account breakdowns or delay which may occur in the system.

An alternative approach rests on the supervisory control theory, which is based on the use of state-transition models, such as finite automata. The supervisory control theory has been developed since the seminal work about 30 years ago (Wonham and Ramadge. 1984; Ramadge and Wonham. 1987). It has become a basic paradigm for the control of discrete event systems (DES). It essentially allows to design a supervisor to prevent conflicts. In an AGV system, it amounts to avoid collisions between vehicles and to avoid blocking situations. It naturally applies to a modeling of AGV systems with finite automata, but a similar approach was proposed in Krogh and Holloway (1991) with Petri net modeling.

The supervisory control theory has a main advantage, it is maximally permissive. Therefore, it gives a maximum freedom degree compatible with the avoidance of conflicts. This permissiveness allows the system to have high flexibility. The supervisor implemented in this paper is designed for operation in real time. Distributed control design for DES in the supervisory control theory (SCT) framework has been studied in Cai and Wonham (2010). The central problem investigated is how to synthesize local controllers for individual independent agents in the context of AGV systems. Another approach has been proposed in Arnaud et al (2009), where the objective function is to minimize the energy expenditure. The main problem is the state-space explosion problem which depends on the number of AGVs in the system. Indeed, in this paper, it is impossible to synthesize a supervisor for four or more AGVs. This problem occurs when the product of the components (AGVs) results in an automaton with a huge number of states.

Compositional or modular approaches can help to overcome the problem of state space explosion. The modular supervisory control offers significant gains in computational complexity (Wonham and Ramadge. 1988; Queiroz and Cury. 2000). In Hill and Tilbury (2006), language projections are used to simplify finite-state machines during synthesis and to construct modular supervisors. To ensure that nonblocking and maximal permissiveness are preserved, the observer property and output-control consistency are imposed as additional requirements on the projection. Another compositional synthesis approach is used (Flordal et al. 2007; Malik and Flordal. 2008; Mohajerani et al. 2011) to remove unnecessary information and to reduce the size of systems. In Flordal et al (2007) and Malik and Flordal (2008), the authors propose automata equivalence for supervisor synthesis, where the synthesis is considered in a nondeterministic setting and leads to some problems when interpreting result and ensuring maximal permissiveness. The supervisor returned may be an over-approximation of the least restrictive solution that is 
not automatically nonblocking. These problems are overcome in Mohajerani et al (2011), where synthesis abstraction is used to abstract automata. The method requires all automata and their abstraction results to be deterministic, which makes some desirable abstraction impossible. Actually, the approach to perform synthesis in several steps as proposed in this paper is described as abstraction and called "halfway synthesis" in the papers cited here. In our case, we do not use any abstraction. We calculate the supervisor using an iterative method and the synthesized previous supervisor. The supervisor is recalculated on-line when a change arises on the configuration of the AGV system.

This paper proposes a solution to the problem of supervisor synthesis using a compositional/incremental method. The specification is expressed in the form of pairs of forbidden states and the supervisor is synthesized on the fly. On our examples, our method allows to reduce the state-space explosion problem, which is well known for the supervisor synthesis.

\subsection{Motivations}

In the industrial world, a circuit is designed to define the traffic lanes of AGVs. These lanes allow to access different storage areas as shown in Fig. 1. Lettered nodes represent intersections and each lane is divided into sections. There are four workstations $(F, H, J$ and $L)$ that represent where AGVs pick up or drop cargo. Nodes $\{A, C, T, Q\}$ represent the entrances of the area and nodes $\{B, D, S, R\}$ the exits of the area.

In the industrial environment (Fig. 1), we can extend this scheme with twenty or more workstations. It then becomes very difficult to manage the traffic in this area to ensure the absence of conflict (see section 2.4) between AGVs. As the number of AGVs wishing to access the area increases, blocking situations are more likely to happen. A blocking situation on an industrial site can stop the production of the company between ten and twenty minutes, which is not admissible for a manufacturer.

\subsection{Our contribution}

In this paper, using a compositional incremental approach, we propose to design the supervisor representing the largest behaviour (or the least restrictive) which is nonblocking and controllable for an AGV system. More precisely, we aim to generate the supervisor and compose intermediate supervisors step by step adding an AGV at every step to build the final supervisor. The synthesized supervisor is the same as the monolithic supervisor of Ramadge and Wonham but its computation is more effective in terms of time and memory. We call it modified monolithic supervisor.

The structure of this paper is as follows. Firstly, in section 2, we present the basic concept of the SCT and the synthesis of supervisor for an AGV system 


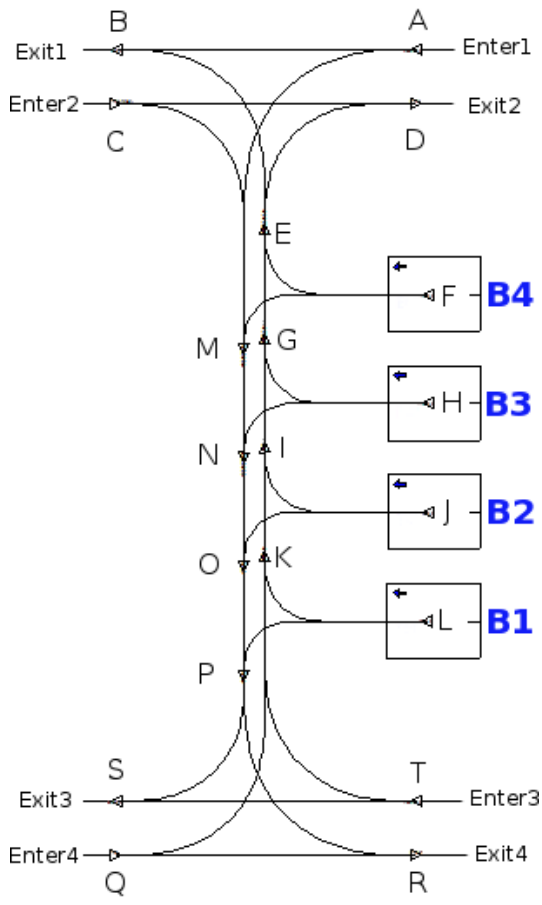

Fig. 1 An example of a storage area.

with forbidden states. In section 3, we propose a compositional approach to synthesize on-line a supervisor, we prove the compositionality and we discuss about the complexity. Finally, in section 4, this approach is applied to real cases from industrial world.

\section{Notations and preliminaries}

This section presents notations and mathematical framework used in this paper. We will consider DES modeled by automata in the context of the SCT put forth by Ramadge and Wonham (Ramadge and Wonham. 1989; Cassandras and Lafortune. 2006).

\subsection{Finite automata}

A discrete event system is a dynamic system in which state changes occur at discrete points in time. They can be represented by an automaton denoted by $G$, which is defined as usually as a five-tuple.

Definition $1 G=\left(Q, \Sigma, \delta, q_{0}, Q_{m}\right)$, where : 
$-Q$ is the finite set of states,

$-\Sigma$ is the finite set of events,

$-\delta: Q \times \Sigma \rightarrow Q$ is the transition function,

$-q_{0} \in Q$ is the initial state,

- $Q_{m} \subseteq Q$ is the set of marked states (final states).

In this paper, all automata are deterministic. Each $\sigma \in \Sigma$ is a label associated with an event. The occurrence of an event corresponds to system changing by passing from one state to another. $\delta(x, \sigma)=y$ means that there is a transition labelled by event $\sigma$ from state $x$ to $y$. In general, $\delta$ is a partial function on its domain. We write $\delta(x, \sigma)$ ! if $\exists y \in Q: \delta(x, \sigma)=y$. Let $\Sigma^{*}$ denote the set of all finite strings $s$ of elements of $\Sigma$, including the empty string, $\epsilon$. For the sake of convenience, $\delta$ is extended from domain $Q \times \Sigma$ to domain $Q \times \Sigma^{*}$ in the following recursive manner:

$$
\begin{aligned}
\delta(x, \epsilon) & =x \\
\delta(x, s \sigma) & =\delta(\delta(x, s), \sigma): s \in \Sigma^{*}, \sigma \in \Sigma .
\end{aligned}
$$

A string $\sigma_{1} \sigma_{2} \ldots \sigma_{n} \in \Sigma^{*}$ is often called a word. We call a valid trace a path from the initial state to a marked state $\left(\delta\left(q_{0}, s\right)=y\right.$ where $s \in \Sigma^{*}$ and $\left.y \in Q_{m}\right)$. We denote $x \stackrel{s}{\rightarrow} y \Leftrightarrow \delta(x, s)=y$ where $x, y \in Q$, as a shorthand. Note that if the states set $Q=\emptyset$ is empty, then $G$ is the empty automaton. The term "empty automaton" refers to an automaton whose state space is empty; an empty automaton necessarily generates and marks the empty set.

In the sequel, we denote $B \backslash A$, the relative complement of $A$ in $B$ where $A$ and $B$ are sets of state. Formally, $B \backslash A=\{x \in B: x \notin A\}$. In other words, the relative complement of $A$ with respect to a set $B$ is the set of elements in $B$ but not in $A$. Then, the notation $\left.\delta\right|_{A}$, means that we are restricting $\delta$ to the domain $A$. Formally, consider the previous automaton $G$, then we have $\left.\delta\right|_{A}(x, \sigma)=y$ iff $\exists y: \delta(x, \sigma)=y$ and $x, y \in A$.

Definition 2 (Language generated and marked)

The language generated by an automaton $G$ is denoted by $L(G)$.

$$
L(G)=\left\{s \in \Sigma^{*}: \delta\left(q_{0}, s\right) !\right\} .
$$

The language marked by $G$ is:

$$
L_{m}(G)=\left\{s \in L(G): \delta\left(q_{0}, s\right) \in Q_{m}\right\} .
$$

The language $L(G)$ represents all the paths that can be followed along the automaton starting from the initial state, while the second language $L_{m}(G)$ is the subset of $L(G)$ (we indeed have $L_{m}(G) \subseteq L(G)$ ) corresponding to paths ending in a marked state and starting from the initial state. The marked language is also called the language recognized by the automaton. Hereinafter we denote that $L=L(G)$ and $L_{m}=L_{m}(G)$. 
Definition 3 (Prefix-closure)

Let $L \subseteq \Sigma^{*}$ a language, its prefix-closure denoted $\bar{L}$, is defined as

$$
\bar{L}=\left\{s \in \Sigma^{*}: \exists t \in \Sigma^{*}(s t \in L)\right\} .
$$

In general, we have $L \subseteq \bar{L}$.

The notation $\bar{L}$ represents the set of all prefixes of string in the language $L$. In the sequel, $|Q|$ denotes the number of states of the set $Q$ and $|\delta|$ denotes the number of transitions of the considered automaton.

\subsection{Operations on automata}

To analyze DES modeled by automata, some operations on automata are used in this paper and presented in this section.

\subsubsection{Accessible part of an automaton}

The accessible part of an automaton is composed of all the states that are accessible or reachable from $q_{0}$ by some words in $L(G)$. When we "delete" a state, we also delete all the transitions that are attached to this state. We denote this operation by $A c(G)$, where $A c$ stands for taking the "accessible" part (Cassandras and Lafortune. 2006). The $A c(G)$ operation is defined as:

$$
\begin{aligned}
A c(G) & =\left(Q_{a c}, \Sigma, \delta_{a c}, q_{0}, Q_{a c, m}\right) \\
Q_{a c} & =\left\{q \in Q: \exists s \in \Sigma^{*}, \delta\left(q_{0}, s\right)=q\right\} \\
Q_{a c, m} & =Q_{m} \cap Q_{a c} \\
\delta_{a c} & =\left.\delta\right|_{Q_{a c} \times \Sigma \rightarrow Q_{a c}}
\end{aligned}
$$

Clearly, the $A c$ operation has no effect on $L(G)$ and $L_{m}(G)$. Thus, from now on, we will always assume, without loss of generality, that an automaton is accessible, that is, $G=A c(G)$.

\subsubsection{Coaccessible part of an automaton}

A state $q$ is said to be coaccessible, if there exists a path in the automaton $G$ from state $q$ to a marked state $q_{m} \in Q_{m}$. We denote the operation of deleting all the states of $G$ that are not coaccessible by $\operatorname{CoAc}(G)$, where $C o A c$ stands for taking the coaccessible part (Cassandras and Lafortune. 2006). This operation is defined as follows:

$$
\begin{aligned}
C o A c(G) & =\left(Q_{\text {coac }}, \Sigma, \delta_{\text {coac }}, q_{0, \text { coac }}, Q_{m}\right) \text { where } \\
Q_{\text {coac }} \subseteq Q & =\left\{q \in Q: \exists s \in \Sigma^{*}, \delta(q, s) \in Q_{m}\right\} \\
q_{0, \text { coac }} & =\left\{\begin{array}{lc}
q_{0} & \text { if } q_{0} \in Q_{\text {coac }} \\
\text { undefined } & \text { otherwise }
\end{array}\right. \\
\delta_{\text {coac }} & =\left.\delta\right|_{Q_{\text {coac }} \times \Sigma \rightarrow Q_{\text {coac }}}
\end{aligned}
$$


If $G=\operatorname{CoAc}(G)$, then $G$ is said to be coaccessible.

\subsubsection{Trim operation}

An automaton which is both accessible and coaccessible is said to be trim (Cassandras and Lafortune. 2006). We define the Trim operation by:

$$
\operatorname{Trim}(G)=\operatorname{CoAc}[A c(G)]=A c[\operatorname{CoAc}(G)]
$$

where the commutativity of $A c$ and $C o A c$ is easily verified. An automaton is nonblocking if every state permits to access to a marked state. A trim automaton is nonblocking. Note that applying only the CoAc operation is sufficient for an automaton to be nonblocking.

\subsubsection{Composition of automata}

A system may be composed of several subsystems. Each subsystem is represented by an automaton. We can design the whole process by the composition of all subsystems.

\section{Definition 4 (Composition of automata)}

Consider two automata $A=\left(Q_{A}, \Sigma_{A}, \delta_{A}, q_{0, A}, Q_{m, A}\right)$ and $B=\left(Q_{B}, \Sigma_{B}, \delta_{B}\right.$, $\left.q_{0, B}, Q_{m, B}\right)$ where $\Sigma_{A}$ and $\Sigma_{B}$ are disjoints. The composition of $A$ and $B$ is the automaton:

$$
A \| B=\left(Q_{A} \times Q_{B}, \Sigma_{A} \cup \Sigma_{B}, \delta_{A B}, q_{0, A B}, Q_{m, A} \times Q_{m, B}\right),
$$

where

- the initial state is $q_{0, A B}=\left(q_{0, A}, q_{0, B}\right)$,

- for all $(x, y) \in Q_{A} \times Q_{B}$ and for all $\left(x^{\prime}, y^{\prime}\right) \in Q_{A} \times Q_{B}$, we have

$$
\delta_{A B}((x, y), \sigma)=\left(x^{\prime}, y^{\prime}\right) \text { iff }\left\{\begin{array}{l}
y=y^{\prime} \text { and } \delta_{A}(x, \sigma)=x^{\prime}, \sigma \in \Sigma_{A} \\
x=x^{\prime} \text { and } \delta_{B}(y, \sigma)=y^{\prime}, \sigma \in \Sigma_{B}
\end{array}\right.
$$

This composition can be generalized to an arbitrary number of automata. It is well known that this operation is associative and commutative.

\subsection{Supervisory control theory}

Supervisory control theory (Wonham and Ramadge. 1984; Ramadge and Wonham. 1987; 1989) permits to design a supervisor from an automaton $M=$ $\left(Q, \Sigma, \delta, q_{0}, Q_{m}\right)$ which represents the system. The set $\Sigma$ is assumed to be separated into two distinct subsets: the set $\Sigma_{c}$ of controllable events and the set $\Sigma_{u}$ of uncontrollable events. This supervisor is an automaton representing the largest behavior for the considered system and respecting the defined specifications. It generates the supremal controllable sublanguage, usually denoted 
$K^{\uparrow}$. Specifications can be defined by a list of forbidden states or a language. Let $M$ be the system. Assume it is not satisfactory, then it must be modified by control. We have to construct a "supervisor" in order to alter the behavior of $M$. We denote $S(M)$ the automaton that represents this supervisor. It generates a sublanguage of $L(M)$, that is $L(S(M))$. The sublanguage corresponding to paths which end in a marked state is denoted $L_{m}(S(M))$. Overall, we have the set inclusions:

$$
\emptyset \subseteq L_{m}(S(M)) \subseteq \overline{L_{m}(S(M))} \subseteq L(S(M)) \subseteq L(M) .
$$

For a better understanding of this article, we recall the concept of controllability defined in (Cassandras and Lafortune. 2006):

\section{Definition 5 (Controllability)}

Let $K$ and $L=\bar{L}$ be languages over event set $\Sigma$. Let $\Sigma_{u}$ be a designated subset of $\Sigma . K$ is said to be controllable with respect to $L$ and $\Sigma_{u}$ if

$$
\bar{K} \Sigma_{u} \cup L \subseteq \bar{K}
$$

By definition, controllability is a property of the prefix-closure of a language. Thus $K$ is controllable if and only if $\bar{K}$ is controllable. For this paper, we need a nonblocking supervisor, the definition is as follows:

Definition 6 (Nonblocking in controlled system)

The DES $S(M)$ is nonblocking if:

$$
L(S(M))=\overline{L_{m}(S(M))} .
$$

We have to extend this definition if we have a set of uncontrollable events in $M$. To deal with uncontrollability, we recall the nonblocking controllability theorem (Cassandras and Lafortune. 2006) :

Theorem 1 (Nonblocking controllability theorem)

Consider the language $K \subseteq L_{m}(M)$ where $K \neq \emptyset$. There exists a nonblocking supervisor $S$ for $M$ such that:

$$
L_{m}(S(M))=K \text { and } L(S(M))=\bar{K}
$$

if and only if the two following conditions hold:

- Controllability: $\bar{K} \Sigma_{u} \cap L(M) \subseteq \bar{K}$

- $L_{m}(M)$-closure: $K=\bar{K} \cap L_{m}(M)$

As it is well-known, the family of controllable and $L_{m}(M)$ - closed sublanguages of $L_{m}(M)$ is closed for the union of languages. As a consequence, if this family is non empty, it contains a supremal element that is a maximally permissive nonblocking supervisor. To build such a supervisor, simply apply the fixpoint procedure of Wonham and Ramadge (1984) (more details in section 2.4.4). Suppress the forbidden states (list of states) and keep the accessible and coaccessible part of the resulting automaton. If the system contains uncontrollable events, we remove also states that lead to a forbidden state through a series of uncontrollable events and apply the Trim operation. 


\subsection{AGV fleet system}

Nowadays, a common approach to ensure that the system is nonblocking, is to define the area like a zone of mutual exclusion which prevents any AGV from getting into the area if an AGV is already inside (only one AGV in the area). We have to define the initial and the final state and only apply the Trim operation. This method ensures to never stop the production process and in some cases it will even maintain an acceptable production rate. However, this is not entirely satisfactory, so we need to make a choice: either we guarantee no blocking and a continuous production or we propose to upgrade this approach, by proposing to allow others AGVs to go inside the area. This permits to increase the production, but brings the risk of blocking situations. Using the supervisory control theory of Ramadge and Wonham, we guarantee no conflict and the most permissive behavior. However, if there are too many AGVs, we can have a long computation time due to a combinatorial explosion (see section 3).

An AGV system is composed of a set of vehicles traveling on a circuit to accomplish various missions. Various tasks consist in delivering or picking-up manufactured part at different locations of the circuit. For the system working correctly, the AGV fleet must be coordinated with a supervisor. The AGV fleet control uses several steps. Firstly, the system must assign different tasks to available AGVs that have no current jobs and are waiting for a mission. This function is not treated in this article, missions are assumed to be already dispatched. Then, the supervisor must define a path for each vehicle on the circuit, avoiding conflicts, to guarantee a secure behavior of the production process. There are two types of conflicts :

- a collision, when two or more vehicles collide.

- a blocking situation, when there is no solution (no path, apart those leading to a collision) to achieve the mission. Fig. 2 illustrates a simple blocking situation between two AGVs.

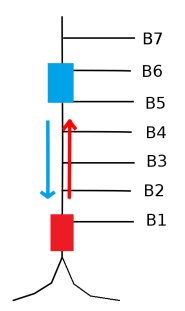

Fig. 2 Blocking situation between 2 AGVs

To optimize the system behavior and allow a fluid circulation of AGVs, the supervisory control theory is a good solution since it will generate the most permissive supervisor. 
2.4.1 Basic model

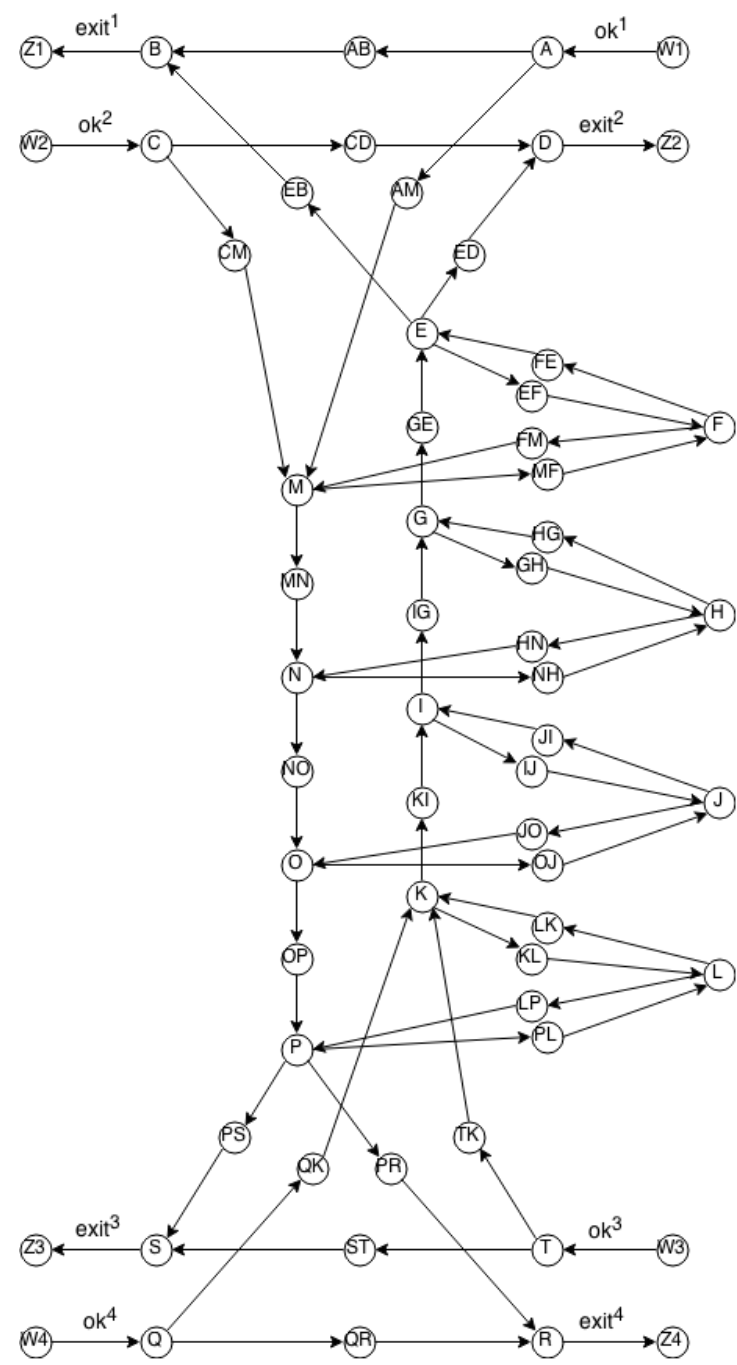

Fig. 3 One AGV's template

A model representing the movements of vehicles on the circuit is designed. Each AGV, which is an autonomous process in the overall process, is represented as a template. The circuit, according to its physical configuration, is partitioned into sections and intersections. Each time an AGV moves in a new intersection or in a new section, a new state in the template is occupied, which 
reflects a change of the model's state. The template associated to the circuit shown in Fig. 1 is represented in Fig. 3 that we denote $T=\left(Q_{T}, \Sigma_{T}, \delta_{T}, \emptyset, \emptyset\right)$, where the initial state and the final state are not defined. It is the physical position of the AGV (resp. its mission) that will determine the initial state (resp. the final state).

For practical reasons, each state that represents a section in the template is named $X Y_{i}$ where $X$ is the beginning of the section, $Y$ the end and $i$ the number of the AGV. For each section, we have two transitions, the first input transition $C_{X Y}$ is controllable and represents the AGV moving on the section between $X$ and $Y$ from $X$ to $Y$, and the second output transition $U_{Y}$ is uncontrollable and represents the AGV arriving at the intersection $Y$. For example, if the location $C M_{1}$ is set, then the $A G V_{1}$ is moving on the section between $C$ and $M$ from $C$ to $M$, we fired the transition $C_{C M}$. The transitions of the different $\mathrm{AGVs}$ are independent, therefore it is necessary to name them differently. The transitions of the AGV number $i$ are denoted $C_{i X Y}$ and $U_{i X Y}$. The different alphabets $\Sigma_{i}$ are separated.

All AGV templates are similar (same states and edges), except for the initial state $q_{0}$ that can be linked to any state according to AGVs positions on the circuit and the final state $q_{m}$ which depends on their mission. This state $q_{0}$ (resp. $q_{m}$ ) allows a dynamic definition of the initial (resp. final) state for each template. Each state in Fig. 3 represents the presence of the AGV on an intersection, a section or a workstation.

In the sequel, we consider a system $M$ with $n$ AGVs that run on the same circuit which is represented by a template as described previously. The automaton $G_{i}=\left(Q_{i}, \Sigma_{i}, \delta_{i}, q_{0, i}, q_{m, i}\right)$ represents the automaton of $A G V_{i}$ on the circuit where $q_{0, i}$ is the initial state (represents the starting position of the AGV on the circuit) and $q_{m, i}$ the final state (the goal of the AGV). Since each AGV moves on the same circuit, we can represent the system as follows :

$$
M=G_{1}\left\|G_{2}\right\| \ldots \| G_{n} .
$$

where $G_{i}$ represents the automaton of $A G V_{i}$, for $i=1$ to $n$. We have $Q_{i}=Q_{T}$ because AGVs move on the same circuit. A state of $M$ is of the form:

$$
\left(x_{1}, x_{2}, \ldots, x_{n}\right) \in Q_{M} .
$$

where $x_{i} \in Q_{T}$, for $i=1$ to $n$, is the position of $A G V_{i}$ on the circuit and the positions $x_{i}$ are arranged in ascending order according to the number of AGV.

\subsubsection{The waiting states and exit states}

In the case of the circuit represented in Fig. 3, the entrances of the area are represented by the states $\{A, C, T, D\}$ whereas the exits are represented by the states $\{B, D, S, R\}$. The states $\{W 1, W 2, W 3, W 4\}$ represent the first physical 
point outside the area and before its associated entrance (e.g. the state $W 1$ is associated to the entrance $A$ ). We name this set of states the waiting states, denoted $\mathbb{W}$, since an $\mathrm{AGV}$ that is in one of these states is waiting for the authorization to go inside the area. Therefore, we define a controllable event $o k_{i}^{j} \in \Sigma_{c}$ and that means the $A G V_{i}$ has the authorization to go inside the area and it is physically at the Entrance $_{j}$. In other words, it means that a supervisor was designed for all AGVs already inside the area and the new AGV. If no solution exists with the new AGV, then it will wait in this waiting state until a solution is found.

The states $\{Z 1, Z 2, Z 3, Z 4\}$ in Fig. 3, represent the first physical point outside the area and after its associated exit (e.g. the state $Z 1$ is associated to the exit $B$ ). We name this set of states the exit states, denoted $\mathbb{E}$. Moreover, we define an uncontrollable event exit ${ }_{i}^{j} \in \Sigma_{u}$ and that means the $A G V_{i}$ has left the area by the Exit ${ }_{j}$. If an AGV has left the area, the supervisor does not care anymore and has to remove it. For example, consider two AGVs where $A G V_{1}$ is in $M$ and $A G V_{2}$ is in $R$ (the system is in the state $\left(M_{1}, R_{2}\right)$ ). $A G V_{2}$ is leaving the area, when it will be in the exit state $Z_{4}$, then the system will be in the state $\left(M_{1}, Z_{4}\right)$. Therefore we have the state equality $\left(M_{1}, Z_{4}\right)=\left(M_{1}, \emptyset_{2}\right)=\left(M_{1}\right)$, since the $A G V_{2}$ is out of the area. Note that all waiting states and exit states are safe, i.e., no collisions can appear with any AGVs (more details in the next section 2.4.3).

\subsubsection{The specification}

Our aim is to avoid blocking situations (see Fig. 2) and thus remove all paths that lead to a blocking situation and that do not permit to reach the final state.

The first step preliminary to the synthesis of a supervision system, consists in the determination of possible conflicts that leads to the specification. A supervisor, we has to express the specifications that must be satisfied. It consists in a list of forbidden states. Unlike most modular approaches, we have a single global specification and not a specification for local agents. This problem is dealt in Komenda et al (2008) for modular systems. The global system is $\bigcap_{i=1}^{n} P_{i}^{-1} L\left(G_{i}\right)$ (where $P_{i}$ is the canonical projection $\bigcup_{i=1}^{n} \Sigma^{*} \rightarrow \Sigma_{i}^{*}$ ) that is a language over $\Sigma=\bigcup_{i=1}^{n} \Sigma_{i}$, and we have only one specification $S p e c \subseteq \Sigma^{*}$.

Each AGV has only one alphabet. In our case, we want AGVs to be alone on a section or an intersection (beginning/end of a section). We need to delete all states (and associated controllable transitions) $q=\left(x_{1}, x_{2}, \ldots, x_{n}\right)$ of the automaton $M$ where:

$$
\exists i, j \in[1, n]: x_{i}=x_{j} .
$$

We say that the states $x_{i}$ and $x_{j}$ are conflicting. Moreover, we say that two states are conflicting if there is a collision between both AGVs. Furthermore, the designer of the circuit has to apply a procedure to add the couple of states where a risk of collision exists. For example, consider the configuration shown 
in Fig. 4 where two AGVs are moving in a area $\left(A G V_{1}\right.$ and $\left.A G V_{2}\right)$. In Fig. 4(a), there is no conflict, both AGVs are in their workstation and $A G V_{2}$ can get out without any problem. Its path is drawn by a series of rectangles representing the physical size of the AGV on the circuit, we note no collision between both AGVs. In contrast, in Fig. 4(b), $A G V_{2}$ must exit through the bottom of the area. By zooming the Fig. 4(b), we see a collision between both AGVs in Fig. 4(c). In accordance with the template automaton $T$, the states $L P$ and $J$ are conflicting for example and many others. The set of forbidden states $\mathcal{I}(T)$ is defined as follows:

$$
\mathcal{I}(T)=\left\{\left(x_{i}, x_{j}\right): x_{i}, x_{j} \text { are conflicting, }\left(x_{i}, x_{j}\right) \in Q_{T} \times Q_{T}\right\}
$$

$\mathcal{I}(T)$ is a list of pairs of states that are conflicting as described above. We generate this list of forbidden states from the template automaton $T$, the shape and the size of the AGVs.

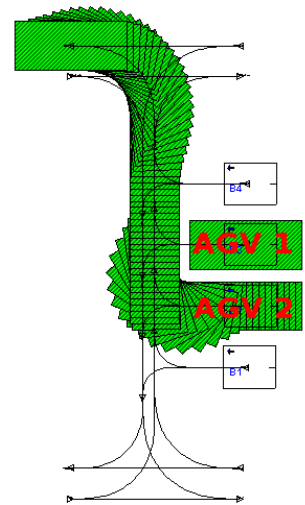

(a) No conflict

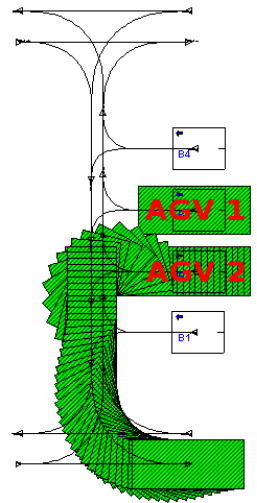

(b) Collision

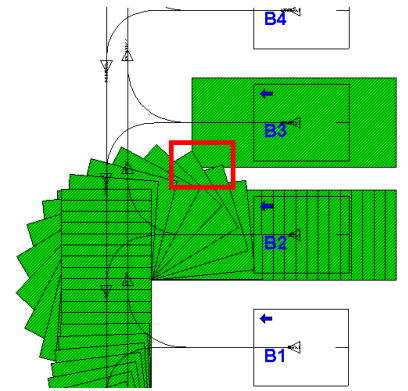

(c) Zoom on collision (b)

Fig. 4 Example of potential conflict

In the previous section, we talked about the safety of waiting states denoted $\mathbb{W}$ and exit states denoted $\mathbb{E}$ of a template automaton. In the sequel, we assume that all waiting states and exit states are safe. We can now formalize this statement as follows.

$$
\forall\left(x_{i}, x_{j}\right) \in \mathcal{I}(T), x_{i}, x_{j} \notin \mathbb{E} \cup \mathbb{W}
$$

where $\mathbb{E} \cup \mathbb{W} \subseteq Q_{T}$. In other words, a waiting state or an exit state can not be conflicting with any states of the template.

\subsubsection{Synthesis of supervisor with forbidden states}

Once we have the specification, we have to synthesize the supervisor. It can be calculated using the standard fixpoint algorithm of the supremal control- 
lable sublanguage (Cassandras and Lafortune. 2006), usually denoted the $\uparrow C$ operation.

Consider the previous automaton $M$, where $M=\left(Q, \Sigma, \delta, q_{0}, Q_{m}\right)$. Let $\Gamma$ : $Q \rightarrow 2^{\Sigma}$ be the active event function where $\Gamma(q)=\{\sigma \in \Sigma: \delta(q, \sigma) !, q \in Q\}$. In words, $\Gamma(q)$ is the set of all events $\sigma$ for which $\delta(q, \sigma)$ is defined. We denote $\Gamma_{M}$ the active event function restricted to the automaton $M$. We present the Standard Algorithm for $\uparrow C$ applied to $M$ where the specification is a list of forbidden states in Algorithm 1.

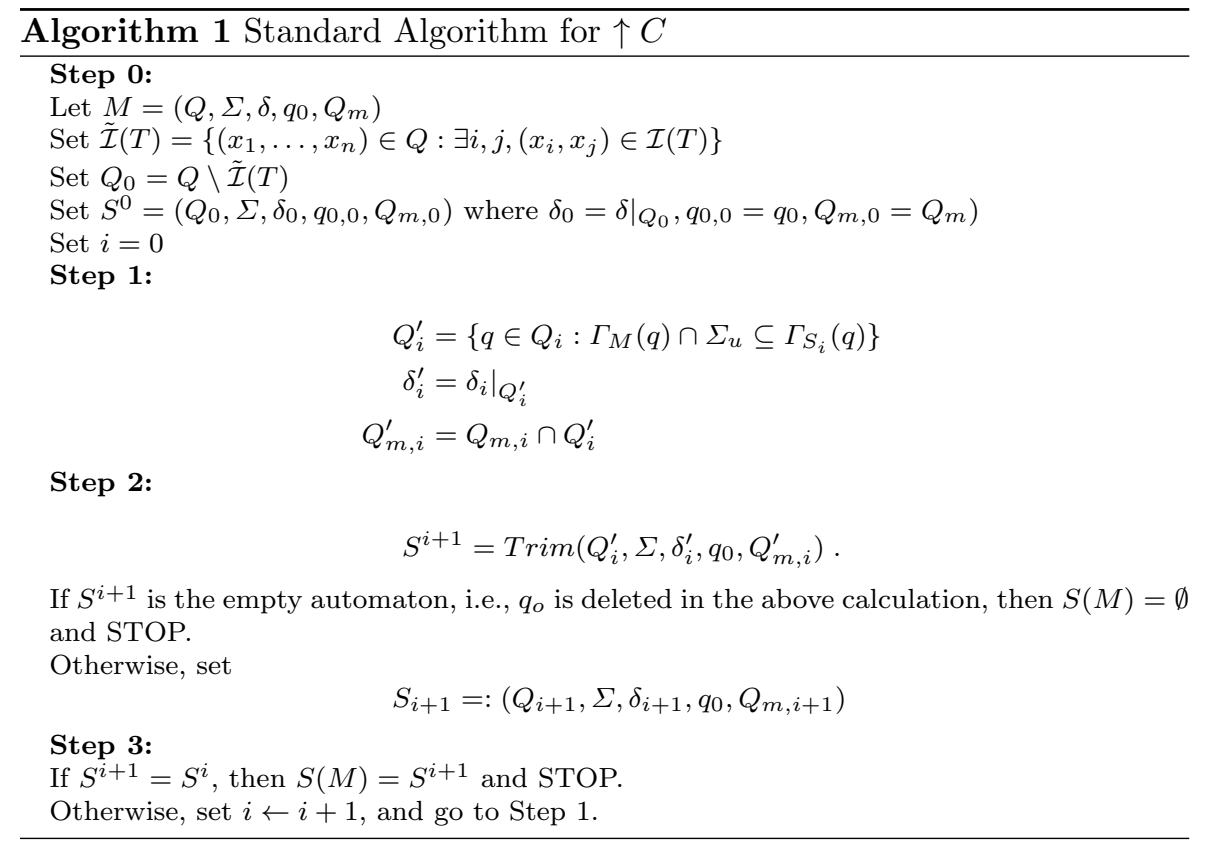

All states removed during the operation are named "bad states" since they can lead to a conflict in the system. The initial Step 0 removes the forbidden states of the set $\mathcal{I}(T)$. The Step 1 removes all uncontrollable states due to controllability issues, i.e., all states that lead to a bad state via a sequence of uncontrollable events. The Step 2 removes all states due to nonblocking issues, i.e., all states that are not coaccessible (nonblocking issues) and not accessible (unnecessary states). Moreover, it checks if we obtain the empty automaton (no solution). Finally, the Step 3 checks the end of the operation. Note that an empty solution, say $S(M)=\emptyset$, means that there exists no supervisor able to coordinate the AGV fleet to ensure the absence of conflict. The necessary and sufficient condition for the existence of a supervisor (and for the admissibility of the considered AGV set) is that $S(M) \neq \emptyset$.

We note that $L(S(M))=\overline{L_{m}(S(M))}$. For example, we consider a fleet of three AGVs moving on the circuit as shown in Fig. 3. This automaton has 54 states and 68 transitions. To obtain the automaton $M$ which represents the 
overall system (all behaviors), we do the composition of the automata $G_{i}, i \in$ $\{1 ; 2 ; 3\}$. The automaton $M$ has $157,464\left(54^{3}\right)$ states and $594,864\left(3 \times 54^{2} \times 68\right)$ transitions. The supervisor $S(M)$ ensures no conflict between AGVs and $S(M)$ is maximally permissive and controllable. If no solution exists, then $S(M)=\emptyset$.

\section{Compositional approach and synthesis of supervisor}

\subsection{Basic idea}

The main difficulty to design such a supervisor is the great number of states and the computation time. Consider the previous system $M$ with $n$ AGVs. The automaton $M$ represents all the behaviors on the circuit, and it has $|Q|^{n}$ states and $n \times|Q|^{n-1} \times|\delta|$ transitions. We easily see that the complexity is exponential and is depending on the number of AGVs. In many cases, if $n \leq 3$ there is no problem, but for $n \geq 4$ we can meet the state-space explosion problem or a long computation time. To reduce this problem, we use a compositional approach. When a new task is assigned to an AGV, we synthesize the new controller from the previous controller and thus we reduce the computational time. This approach reduces the intermediate state-space, it is maximally permissive and may be faster than the standard approach (monolithic approach). We synthesize a new supervisor step by step, where each step adds a new AGV and compose it with the previous supervisor.

For example, consider a system with four AGVs modeled by $G_{1}, G_{2}, G_{3}$ and $G_{4}$, we have $M=G_{1}\left\|G_{2}\right\| G_{3} \| G_{4}$. Let $S(M) \subseteq M$, the supervisor of $M$. We will demonstrate that:

$$
\begin{aligned}
S(M) & =S\left(S\left(S\left(S\left(G_{1}\right) \| G_{2}\right) \| G_{3}\right) \| G_{4}\right) \\
& =S\left(S\left(G_{1} \| G_{2}\right) \| S\left(G_{3} \| G_{4}\right)\right) \\
& =S\left(G_{1}\left\|G_{2}\right\| G_{3} \| G_{4}\right) .
\end{aligned}
$$

\subsection{Compositional synthesis}

Consider a system with $n$ AGVs and the automaton $G_{i}=\left(Q_{i}, \Sigma_{i}, \delta_{i}, q_{0, i}, Q_{m, i}\right)$ that represents the i'th $A G V$ where $i \in[1, n]$. Let $S_{G}(M)=\left(Q_{S_{G}(M)}, \Sigma_{S_{G}(M)}\right.$, $\left.\delta_{S_{G}(M)}, q_{0, S_{G}(M)}, Q_{m, S_{G}(M)}\right)$ be the supervisor obtained with the monolithic approach. Let $S_{C}(M)=\left(Q_{S_{C}(M)}, \Sigma_{S_{C}(M)}, \delta_{S_{C}(M)}, q_{0, S_{C}(M)}, Q_{m, S_{C}(M)}\right)$ be the supervisor obtained with the compositional approach as described in Fig. 5(a). The synthesis algorithm is Algorithm 2.

We obtain:

$$
\begin{aligned}
S_{C}(M) & =S\left(S\left(S\left(\ldots S\left(S\left(G_{1}\right) \| G_{2}\right) \| \ldots\right) \| G_{n-1}\right) \| G_{n}\right) \\
& =S\left(S\left(S\left(\ldots S\left(G_{1} \| G_{2}\right) \| \ldots\right) \| G_{n-1}\right) \| G_{n}\right)
\end{aligned}
$$

The second equality is equivalent since $S\left(G_{1}\right)$ represents the supervisor for only one AGV, therefore, there is no conflict and $S\left(G_{1}\right)=G_{1}$. 


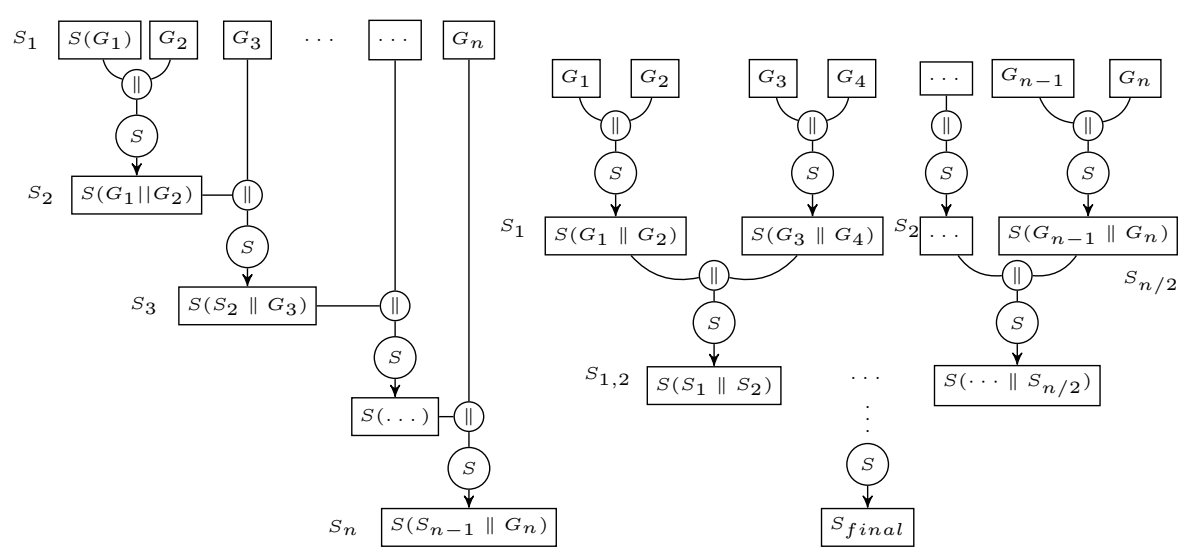

(a) $S_{k+1}$ from $S_{k}$

(b) Synthesis in pairs

Fig. 5 Compositional synthesis

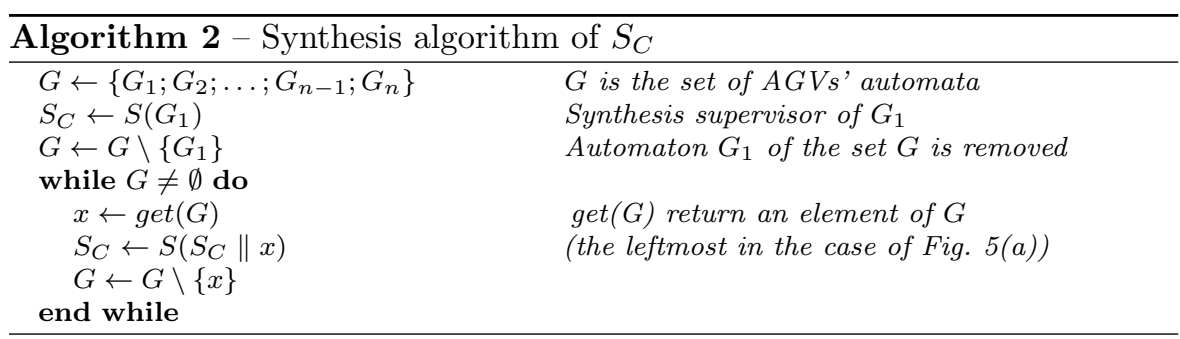

Theorem $2 S_{G}(M)=S_{C}(M)$.

Proof For this proof, we will denote $S_{C}(M)=S_{C}$ and $S_{G}(M)=S_{G}$ as shorthand. To prove this theorem, we have to demonstrate that $S_{C}$ and $S_{G}$ have equal state sets, alphabets, transition relations, initial states, and marked state sets. By definition of the composition, $S_{C}$ and $S_{G}$ have the same initial state and the same alphabet. We will first prove that they have the same state set (potentially the empty set). By definition, the monolithic supervisor $S_{G}$ is sound and complete. We will prove that the compositional supervisor $S_{C}$ is sound (i.e. $Q_{S_{C}} \subseteq Q_{S_{G}}$ ) and complete too (i.e. $Q_{S_{C}} \supseteq Q_{S_{G}}$ ). We will denote $S_{k}=\left(Q_{S_{k}}, \Sigma_{S_{k}}, \delta_{S_{k}}, q_{0, S_{k}}, Q_{m, S_{k}}\right)$ the compositional supervisor for the first $k$ AGVs where $S_{k+1}=S\left(S_{k} \| G_{k+1}\right)$ (by definition of the compositional synthesis) and $k \in[1, n]$. Moreover, consider two automata $A$ and $B$, we will denote $Q_{A \| B}$, the state set of the automaton $A \| B$. Let $k \in[1, n]$ where $n$ is the number of AGVs. We define the following projection $P_{k}: Q_{M} \longrightarrow Q_{1} \times \cdots \times Q_{k}$ :

$$
P_{k}\left(x_{1}, \ldots, x_{n}\right)=\left(x_{1}, \ldots, x_{k}\right) .
$$

where $P_{k}(q)$ represents the $k$ first states of $q$, and consequently, the states of the $k$ first AGVs. 
1. The soundness

By definition of the synthesis of supervisor (see section 2.4.4), the compositional supervisor $S_{C}$ is sound since it removes all bad states and is nonblocking. Therefore we have the following inclusion: $Q_{S_{C}} \subseteq Q_{S_{G}}$.

2. The completeness

Now, we need to prove that $S_{C}$ is complete, i.e., $Q_{S_{C}} \supseteq Q_{S_{G}}$. We will prove this result by induction on the number of AGVs $k \in[1, n]$. We have to show that for every state $q=\left(x_{1}, \ldots, x_{n}\right) \in Q_{S_{G}}$, the part of $q$ $P_{k}(q)=\left(x_{1}, \ldots, x_{k}\right) \in Q_{S_{k}}$ (for the rest of the proof, we denote $q^{k}=P_{k}(q)$ as shorthand). In other words, for every state $q$ of the monolithic supervisor $S_{G}$, the part $q^{k}$ of $q$ is a state of the compositional supervisor $S_{k}$ thus every state $q$ of $S_{G}$ is a state of $S_{n}=S_{C}$. Formally, we have to prove:

$$
\forall q \in Q_{S_{G}}, \forall k \in[1, n], q^{k} \in Q_{S_{k}}
$$

Clearly, nothing is to be shown for $k=1$ as $S_{1}=G_{1}$ contains all the possible states $x_{1}$ as discussed previously. So, $q^{1}=\left(x_{1}\right) \in Q_{S_{1}}$.

Now, assume the claim (1) has been shown for some $k \geq 1$ (it means that for every state $q=\left(x_{1}, \ldots, x_{n}\right)$ of $S_{G}$, it holds that $q^{k}=\left(x_{1}, \ldots, x_{k}\right)$ is a state of $\left.S_{k}\right)$. Then, we have to show that this holds true for $k=k+1$, and so, $q^{k+1} \in Q_{S_{k+1}}$.

By definition, $x_{k+1} \in G_{k+1}$. So, $q^{k+1}=\left(x_{1}, \ldots, x_{k+1}\right) \in Q_{S_{k} \| G_{k+1}}$. Therefore, it remains to be shown that this state survives to the synthesis operation $S\left(S_{k} \| G_{k+1}\right)$. It will be shown that for every state $q \in Q_{S_{G}}$, the part $q^{k+1}$ is contained in every iteration of the synthesis fixpoint algorithm $S^{l}\left(S_{k} \| G_{k+1}\right)$ (for $l \geq 0$ ), thus for every state $q, q^{k+1}$ is contained in $S\left(S_{k} \| G_{k+1}\right)$. We will prove the following formula by induction on $l$.

$$
\forall q \in Q_{S_{G}}, \forall l \in \mathbb{N}, q^{k+1} \in Q_{S^{l}\left(S_{k} \| G_{k+1}\right)}
$$

First, we prove this for $l=0$. It is clear that $q^{k+1} \in Q_{S^{0}\left(S_{k} \| G_{k+1}\right)}$ since the state $q \in Q_{S_{G}}$ is a state of the monolihic supervisor and therefore, it is not a forbidden state. We recall that forbidden states are only removed during the Step 0 of the synthesis fixpoint algorithm (see section 2.4.4). As the state $q$ is not a forbidden state, thus none of the pairs $\left(x_{i}, x_{j}\right)$ is a conflict, i.e., $\forall x_{i}, x_{j} \in q,\left(x_{i}, x_{j}\right) \notin \mathcal{I}(T)$. So, the part $q^{k+1}$ also is not a forbidden state.

Now assume the claim (2) has been shown for some $l \geq 0$ (i.e. every state $q=\left(x_{1}, \ldots, x_{n}\right) \in Q_{S_{G}}$, the part $q^{k+1}=\left(x_{1}, \ldots, x_{k+1}\right)$ is a state of $\left.S^{l}\left(S_{k} \| G_{k+1}\right)\right)$. Then, we have to show that this holds true for $l=l+1$, and so, $q^{k+1} \in Q_{S_{k+1}}$.

To show that $q^{k+1}$ is contained in $S^{l+1}\left(S_{k} \| G_{k+1}\right)$, we have to show that the state is never removed during this synthesis step. There is only two ways to remove a state during the synthesis: (i) due to the controllability (Step 1 of the synthesis algorithm, section 2.4.4), if a state may lead to a bad 
state via a sequence of uncontrollable events, (ii) due to the nonblocking issues (Step 2 of the synthesis algorithm, section 2.4.4), if a state is not coaccessible.

(i) For controllability, assume $\delta_{S_{k+1}}\left(q^{k+1}, u\right)=\left(y_{1}, \ldots, y_{k+1}\right)$ for some $u \in$ $\Sigma_{u}^{*}$ such that $\delta_{S_{k+1}}\left(q^{k+1}, u\right)$ !. Then, we have $\delta_{S_{G}}(q, u)=\left(y_{1}, \ldots, y_{k+1}\right.$, $\left.x_{k+2}, \ldots, x_{n}\right)$ since the $G_{i}$ do not share events. As $q=\left(x_{1}, \ldots, x_{n}\right) \in$ $Q_{S_{G}}$, and $S_{G}$ is controllable, it follows that $\left(y_{1}, \ldots, y_{k+1}, x_{k+2}, \ldots, x_{n}\right) \in$ $Q_{S_{G}}$. By inductive assumption, the state $\left(y_{1}, \ldots, y_{k+1}\right) \in Q_{S^{l}\left(S_{k} \| G_{k+1}\right)}$.

As this holds for all $u \in \Sigma_{u}^{*}$, by definition of synthesis (section 2.4.4), this means that $q^{k+1} \in Q_{S^{l+1}\left(S_{k} \| G_{k+1}\right)}$ as far as controllability is concerned.

(ii) For nonblocking, we recall that $q \in Q_{S_{G}}, S_{G}$ is nonblocking. Therefore, there exists a path in $S_{G}$ from $q=\left(x_{1}, \ldots, x_{n}\right)$ to a marked state:

$$
\left(x_{1}, \ldots, x_{n}\right) \stackrel{\sigma_{1}}{\longrightarrow}\left(x_{1}^{1}, \ldots, x_{n}^{1}\right) \stackrel{\sigma_{2}}{\longrightarrow} \ldots \stackrel{\sigma_{p}}{\longrightarrow}\left(x_{1}^{p}, \ldots, x_{n}^{p}\right)
$$

where all the states $\left(x_{1}^{r}, \ldots, x_{n}^{r}\right) \in Q_{S_{G}}$ with $0 \leq r \leq p$, and the end state is a marked state, i.e., $\left(x_{1}^{p}, \ldots, x_{n}^{p}\right) \in Q_{m, S_{G}}$. By inductive assumption, we have the parts $\left(x_{1}^{r}, \ldots, x_{k+1}^{r}\right) \in Q_{S^{l}\left(S_{k} \| G_{k+1}\right)}$. Since the $G_{i}$ do not share events, the above path can be reduced by removing events not in $G_{1}, \ldots, G_{k+1}$ to get a path from $q^{k+1}=\left(x_{1}, \ldots, x_{k+1}\right)$ to a marked state:

$$
\left(x_{1}, \ldots, x_{k+1}\right) \stackrel{\tilde{\sigma}_{1}}{\longrightarrow}\left(x_{1}^{1}, \ldots, x_{k+1}^{1}\right) \stackrel{\tilde{\sigma}_{2}}{\longrightarrow} \ldots \stackrel{\tilde{\sigma}_{p}}{\longrightarrow}\left(x_{1}^{p}, \ldots, x_{k+1}^{p}\right)
$$

where the end state is a marked state in $G_{1}\|\cdots\| G_{k+1}$ and the notation " " means "restricted to the automaton $G_{1}\|\cdots\| G_{k+1}$ ". As all the states on the above path are in $S^{l}\left(S_{k} \| G_{k+1}\right)$, it follows by definition of synthesis that $q^{k+1} \in Q_{S^{l+1}\left(S_{k} \| G_{k+1}\right)}$ as far as nonblocking is concerned.

Thus, the state $q^{k+1}$ is not removed due to controllability nor due to nonblocking, so by definition of synthesis, the state $q^{k+1}=\left(x_{1}, \ldots, x_{k+1}\right) \in$ $Q_{S^{l+1}\left(S_{k} \| G_{k+1}\right)}$.

This proves the inclusion $Q_{S_{C}} \supseteq Q_{S_{G}}$, and therefore, since the reverse inclusion was already verified, $S_{C}$ and $S_{G}$ have exactly the same set of states. In other words, $Q_{S_{G}}=Q_{S_{C}}$ and $S_{C}$ is complete.

We now prove that $S_{G}$ and $S_{C}$ have the same transitions. Let $q=\left(x_{1}, \ldots, x_{n}\right)$ and $q^{\prime}=\left(x_{1}^{\prime}, \ldots, x_{n}^{\prime}\right)$ be two states of $Q_{S_{G}}=Q_{S_{C}}$. By definition of the composition (Definition 4), we have a transition between $q$ and $q^{\prime}$ if and only if $\exists i \in[1, n], \exists \sigma \in \Sigma_{i}$ such that $\delta_{G_{i}}\left(x_{i}, \sigma\right)=x_{i}^{\prime}$ and $\forall j \in[1, n], j \neq i \Rightarrow x_{j}=x_{j}^{\prime}$. Hence we have $\delta_{S_{C}}(q, \sigma)=\delta_{S_{G}}(q, \sigma)=q^{\prime}$. Therefore $\delta_{S_{C}}=\delta_{S_{G}}$. We have finally checked the equalities: $Q_{S_{C}}=Q_{S_{G}}, \Sigma_{S_{C}}=\Sigma_{S_{G}}, \delta_{S_{C}}=\delta_{S_{G}}, q_{0, S_{C}}=$ $q_{0, S_{G}}, Q_{m, S_{C}}=Q_{m, S_{G}}$ that ends the proof. 
Let us denote $\otimes$ the operator of supervisor design for two automata: $G_{i} \otimes$ $G_{j}=S\left(G_{i} \| G_{j}\right)$. This operator is commutative, since the composition $\|$ is commutative too:

$$
G_{1} \otimes G_{2}=G_{2} \otimes G_{1}
$$

The operator $\otimes$ is also associative, we obtained that:

$$
\begin{aligned}
S\left(G_{1}\left\|G_{2}\right\| G_{3}\right) & =S\left(S\left(G_{1} \| G_{2}\right) \| G_{3}\right) \\
& =S\left(G_{1} \| S\left(G_{2} \| G_{3}\right)\right)
\end{aligned}
$$

which shows that:

$$
\left(G_{1} \otimes G_{2}\right) \otimes G_{3}=G_{1} \otimes\left(G_{2} \otimes G_{3}\right)
$$

As a consequence, the compositional synthesis method can be applied for every order chosen to compose subsystems. Every binary tree generated from the set of AGVs leads to the definition of a compositional synthesis resulting in a supervisor that actually coincides with the monolithic supervisor $S_{G}(M)$.

For instance, let us consider the alternative compositional synthesis, illustrated in Figure 5(b). The figure must be viewed as a tree that should be as balanced as possible. We note $S_{A C}(M)$ the supervisor synthesized with alternative compositional approach. We have $S_{A C}(M)=S\left(S\left(\ldots S\left(G_{1} \| G_{2}\right) \|\right.\right.$ $\left.\ldots S\left(S\left(G_{n-3} \| G_{n-2}\right) \| S\left(G_{n-1} \| G_{n}\right)\right) \ldots\right)$. Thus, we have the following property.

Corollary $1 S_{G}(M)=S_{A C}(M)$.

As said in the introduction, our method is a modified monolithic supervisor because it generates the same language as the standard monolithic supervisor. Every compositional supervisor is actually equal to $S_{G}(M)$, so it is maximally permissive. This is particularly the case of $S_{C}(M)$ and $S_{A C}(M)$. In the case of an AGV system, one needs to have a centralized supervisor but the computation is "modular". It is necessary to have only one supervisor because AGVs move on the same physical part of a circuit, therefore a centralized supervisor allows to avoid conflicts between AGVs. The on-line application of this method is its main advantage (because a new AGV may arrive at any time in the critical area) without having to rebuild the supervisor from scratch (as described in section 3.2).

\subsection{On-line synthesis supervisor}

The proposed compositional approach offers three advantages. The first one is a reduction of the intermediate state-space (necessary during the various steps of synthesis) and thus (in our experiments) the computation time. The second one is the possibility to design intermediate supervisors in any order. 
It always produces the maximally permissive and controllable supervisor. The construction order may be designed to minimize the total computation time, which goes beyond the aim of the present paper.

The main advantage of this approach is to be especially suitable for realtime systems. Indeed, there is no need to recompute the supervisor if a new AGV arrives in the area. Changing the initial state of the current supervisor is enough in order to maintain its accessible and coaccessible parts and to combine it with the automaton of the new AGV. The new initial state is the state in which the system is located at the time of introduction of the new AGV. Thus the computation gain may be significant according to the progress in which the system is. If the first AGVs have performed a large part of their mission, then the computation time will be significantly reduced, and vice versa. Then the computation time depends on the progress of various missions. This property is essential for a real-time system. This approach allows to design a supervisor for a large number of AGVs whereas with a standard approach, we cannot model such a supervisor for five or more AGVs. We have to define a new function to change the initial state of an automaton:

\section{Definition 7 (NIS - New Initial State)}

Let $A=\left(Q, \Sigma, \delta, q_{0}, Q_{m}\right)$ be an automaton. Let $q_{0}^{\prime}$ be the desired new initial state of $A$.

$$
N I S\left(A, q_{0}^{\prime}\right)=\left(Q, \Sigma, \delta, q_{0}^{\prime}, Q_{m}\right)
$$

In other words, $N I S\left(A, q_{0}^{\prime}\right)$ returns an isomorphic automaton to $A$ with the new initial state $q_{0}^{\prime}$.

For example, consider a critical area with $n$ AGVs, it is assumed that $n$ AVGs are moving in the area and they execute instructions sent by the supervisor $S_{n}$ (corresponding supervisor for $n$ AGVs) and a new AGV $\left(A G V_{n+1}\right)$ arrives (enters the area). Let $G_{n+1}$ be the automaton for the $A G V_{n+1}$. Now, we need to change the initial state of $S_{n}$. Let $q_{c}$ the current state of $S_{n}$ when the $A G V n+1$ arrives. Let $q_{c}$ be the current state where the system (of supervisor $\left.S_{n}\right)$ is. The on-line synthesis of the new supervisor is:

$$
S_{n+1}=S\left(\operatorname{Trim}\left(N I S\left(S_{n}, q_{c}\right)\right) \| G_{n+1}\right)
$$

This supervisor is the compositional supervisor $S_{c}$ where $q_{c}$ is the new initial state. As shown in the Theorem 2, this supervisor is nonblocking, controllable and maximally permissive.

\subsection{Entrance of an area}

In this section, we discuss about entrances of an area and particularly on the meaning of a waiting state. When an AGV is in a waiting state, the AGV is outside the area but it has to go inside the area to accomplish its mission. However, if an AGV is in this state, it means that the system has no founded 
a solution without conflict which authorizes it to go inside the area, i.e., the supervisor synthesized with this new AGV is empty and the system keep its old supervisor. Therefore, an AGV which is in a waiting state has to wait in this place until a nonblocking and without conflict supervisor be found. Once such a supervisor is synthesized, the $A G V$ has the authorization to go inside the area and the system can fire the $o k$ transition as discussed in the section 2.4.1. It is important to recall that such a state is a free-conflict state. A collision with an AGV which is in a waiting state is impossible.

\subsection{Exit of an area}

In this section, we discuss about exits of an area and particularly on the meaning of an exit state. When an AGV leaved the area, the AGV fired an exit transition and it is in an exit state. As we said previously, an AGV which is in a exit state is out of the area and we need to remove all elements of this AGV from the supervisor states. The first step preliminary to the deletion consists to apply the NIS and Trim operation to keep only the new accessible part of the system. Consider the system $M$ described in the section 2.4.1 which is supervised by the supervisor $S(M)$. Let $r$ be the AGV number which leaved the area, where $1 \leq r \leq n$. Then, $A G V_{r}$ is in an exit state $x_{r} \in \mathbb{E}$ and the system is in a state $\mathbf{x}$ which is of the form:

$$
\left(x_{1}, \ldots, x_{r}, \ldots, x_{n}\right) .
$$

We apply the NIS operation where $\mathbf{x}$ is the new initial state. Then, we have the new supervisor $S^{\prime}(M)=\operatorname{Trim}(N I S(S(M), \mathbf{x}))$. The second step consists to remove all elements of $A G V_{r}$ from all states of $S^{\prime}(M)$. Let $q \in Q_{S^{\prime}(M)}$ be a state of the new supervisor. After the NIS and Trim operation, no transition can be fired for $A G V_{r}$ since an exit state has no output transition. Therefore, we have to apply Algorithm 3. The AGV number $r$ has been removed from the supervisor, and then the systems contains $n-1 \mathrm{AGVs}$ in the area.

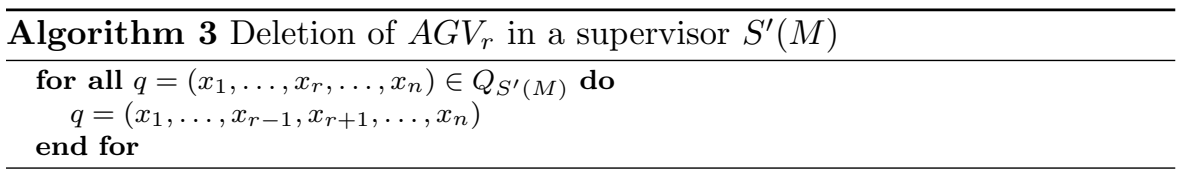

\subsection{A simple example}

We present, in this section, a simple example to illustrate the results of the previous section. The "template" automaton $T$ is shown in Fig. 6.

Two AGVs are moving on the area, $A G V_{1}$ has to go to workstation $W S_{1}$ and $A G V_{2}$ has to go to workstation $W S_{2}$ (Fig. $7(\mathrm{a})$ ). Let $G_{1}$ be the automaton 


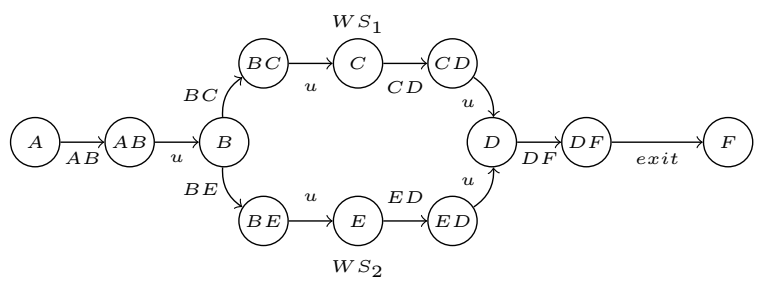

Fig. 6 "Template" automaton $T$

of $A G V_{1}$ and $G_{2}$ be the automaton of $A G V_{2}$. Let $S_{2}=S\left(G_{1} \| G_{2}\right)$. We recall that when an $\mathrm{AGV}$ is in the state $F$ then the $A G V$ has left the area, therefore it is removed from the supervisor and we have $n-1$ AGVs in the area. The $A G V_{3}$ arrives when the $A G V_{1}$ is in the place $D$ and the $A G V_{2}$ is in $W S_{2}$. The $A G V_{3}$ has to go in $W S_{2}$. Let $G_{3}$ be the automaton of the $A G V_{3}$. If we apply the compositional approach described previously, then we have to compose the automaton $G_{3}$ of the $A G V_{3}$ to the supervisor $S_{2}$ of the $A G V_{1}$ and $A G V_{2}$ with the new initial state of $S_{2}$. Let us assume that the new initial state named $q$ where $q=(D, E): D \in Q_{1}, E \in Q_{2}$ and $q \in Q_{1} \times Q_{2}$. Therefore the new supervisor for the three AGVs is as follows:

$$
S_{3}=S\left(\operatorname{Trim}\left(N I S\left(S_{2}, q\right)\right) \| G_{3}\right)
$$

where $\operatorname{Trim}\left(N I S\left(S_{2}, q\right)\right)$ is shown in Fig. 8. The Table 1 shows the states of the automaton of the Fig. 8. Note that states denoted $\emptyset_{i}$ represent that the $A G V_{i}$ is out of the area as explained in the section 2.4.1. The list of forbidden states is generated as described on section 2.4.4. We note that the following pairs of states are forbidden states: $\{(B C, B E),(C D, E D)\} \subset \mathcal{I}(T)$.

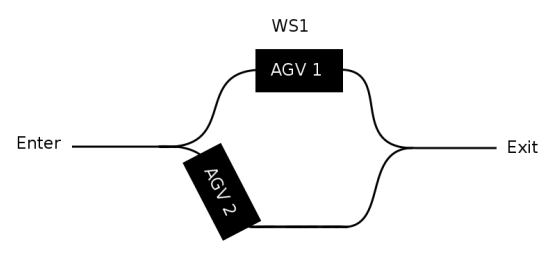

WS2

(a) 2 AGVs moving

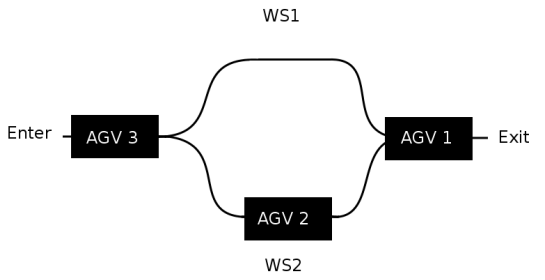

(b) $A G V_{3}$ arrives

Fig. 7 Straightforward example

The automaton $\operatorname{Trim}\left(N I S\left(S_{2}, q\right)\right)$ has 9 states and 10 transitions. At this step, we do not need to recalculate the composition with $\operatorname{Trim}\left(\operatorname{NIS}\left(G_{1}, q_{1}\right)\right)$ and $\operatorname{Trim}\left(N I S\left(G_{2}, q_{2}\right)\right.$ ), as in the monolithic approach, where $q_{1} \in Q_{1}$ (resp. $\left.q_{2} \in Q_{2}\right)$ is the new initial state of $G_{1}$ (resp. $G_{2}$ ). Formally, for the monolithic approach, we have $\operatorname{Trim}\left(N I S\left(G_{1}, q_{1}\right)\right) \| \operatorname{Trim}\left(N I S\left(G_{2}, q_{2}\right)\right)$ and the result 


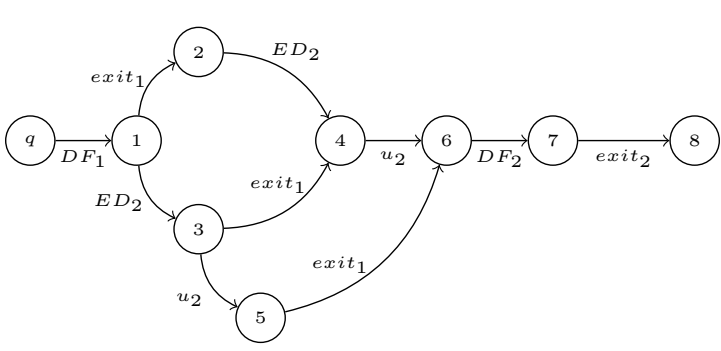

\begin{tabular}{|c|c|}
\hline$q$ & $\left(E_{1}, D_{2}\right)$ \\
\hline 1 & $\left(D F_{1}, E_{2}\right)$ \\
\hline 2 & $\left(\emptyset_{1}, E_{2}\right)$ \\
\hline 3 & $\left(D F_{1}, E D_{2}\right)$ \\
\hline 4 & $\left(\emptyset_{1}, E D_{2}\right)$ \\
\hline 5 & $\left(D F_{1}, D_{2}\right)$ \\
\hline 6 & $\left(\emptyset_{1}, D_{2}\right)$ \\
\hline 7 & $\left(\emptyset_{1}, D F_{2}\right)$ \\
\hline 8 & $\left(\emptyset_{1}, \emptyset_{2}\right)$ \\
\hline
\end{tabular}

Fig. $8 \operatorname{Trim}\left(N I S\left(S_{2}, q\right)\right)$

Table 1 States of Fig. 8

of this operation is an automaton with 15 states and 18 transitions. At this step, we clearly see the gain for the intermediate space used. The compositional method uses the previous supervisor and previous forbidden states have already been removed whereas the monolithic approach rebuilds the previous forbidden states which were removed. The supervisor shown in Fig. 8, is the supervisor when the third AGV arrives at the input of the area. Then, we have to compose it with $G_{3}$ (the automaton of the $A G V_{3}$ ). The final supervisor $S_{3}$ has 47 states and 84 transitions.

3.7 Complexity of monolithic and compositional controller synthesis for AGV

This section estimates the complexity of our on-line compositional synthesis and compares it with the monolithic one. We describe the complexity in terms of generator $M$ and a sublanguage $K$ of $L(M)$. $M$ has $m$ states and $K$ has $n$ states. Consider the problem of synthesizing a supervisor so that the closed loop behavior is a prescribed language $K$. In our case $K$ is not controllable and $K \subseteq L(M)$, therefore the computation of $K \uparrow$ (the largest controllable sublanguage) is of time complexity $O\left(m^{2} n^{2}\right)$ and can be computed in polynomial time (Ramadge and Wonham. 1989). The complexity for the computation of $M=\|_{i=1}^{p} G_{i}$, if each $G_{i}$ has $k$ states, then $M$ has $k^{p}$ states. Therefore the complexity to synthesize a system of $p$ AGVs is $O\left(k^{2 p} n^{2}\right)$. The complexity depends on the number of AGVs and on the size of the system. For the compositional approach, we perform $p-1$ times the operation to synthesize the supervisor $S\left(K^{\uparrow}\right)$. Then we can decompose the complexity for $p$ AGVs as follows: $O\left(k^{2 \times 2} n^{2}\right)+O\left(k^{3 \times 2} n^{2}\right)+\cdots+O\left(k^{2 p} n^{2}\right)=O\left(k^{2 p} n^{2}\right)$. Then the compositional approach does not increase the theoretical complexity to synthesize a supervisor.

In practice we may have a large gain in terms of time and intermediate space. It depends on the topology of the circuit and therefore on the number of forbidden and bad states. If there are many forbidden states, the gain will be important. Indeed, in this case, the size of intermediate supervisors will be significantly reduced. If a new $\mathrm{AGV}$ arrives, numbered $p+1$, as we said previously, we set the initial state of $S_{p}$, apply Trim operation to $S$ and synthesize 
the new supervisor $S_{p+1}$ from $S_{p}$. Assume that $\operatorname{Trim}\left(N I S\left(S_{p}, q\right)\right.$ ) (where $q$ is the new initial state) has $m^{\prime}$ states and $G_{p+1}$ has $k$ states. The complexity for the compositional approach for the step $p+1$ is $O\left(\mathrm{~km}^{\prime 2} \mathrm{n}^{2}\right)$ where $\mathrm{m}^{\prime} \leq m$ because the Trim operation cannot increase the size of an automaton. Moreover, $m^{\prime}$ may be much lower than $m$, it depends on the progress of the system. Let $M=\|_{i=1}^{p} N I S\left(G_{i}, q_{i}\right)$ be the composition of the first $p$ AGVs in the monolithic approach where $q_{i}$ is the new initial state of $G_{i}$. Assume that $M$ has $m^{\prime \prime}$ states, therefore we have $m^{\prime \prime} \geq m^{\prime}$. The complexity at the step $p+1$ for the monolithic approach is $O\left(\mathrm{~km}^{\prime \prime 2} \mathrm{n}^{2}\right)$ where $\mathrm{m}^{\prime \prime}$ may be much larger than $\mathrm{m}^{\prime}$. For the monolithic approach, $m^{\prime \prime}$ is the result of $p$ composition operations whereas, for the proposed approach, we have only one composition operation. This operation may be very costly because it depends directly on the number of AGVs and it is exponential. We clearly see the gain for an on-line synthesis when a new AGV has to go in the area.

For the compositional approach, forbidden states are never built and therefore some paths generated with the monolithic approach will never be generated in our approach. As we said previously, the synthesis is associative, then the order of generation of intermediate supervisors is not important but the time execution and intermediate space may depend on this order. For example, if an $A G V_{1}$ and an $A G V_{2}$ have many forbidden states then it is preferable to generate this supervisor first. Of course, this order should respect the arrival order of the vehicles and in this case, we can not choose the order.

\subsection{Optimization for successive missions.}

To optimize the paths of AGVs, it is essential to know where the AGV will go after completing its mission. We consider in this section that we know the exit of the AGV which often happens. For example, when the mission of an AGV is to move an object from one point to another outside the area. If we treat this problem by considering successively two separate missions for the AGV (one is ending in the area and the other one finishes outside the area), depending on the physical configuration of the circuit, it is possible to encounter situations where two AGVs have completed their first mission, one of the AGV is forced to retreat to let out another. Therefore, the path of the AGV is not optimal.

To solve this problem, it is no longer question to find a supervisor to ensure reachability for a final state but it is a more complex problem. Let $G_{T}$ be the template automaton. Let $q_{0}$ be the initial state. Let $q_{1}$ be the state to be reached for its first mission, and $q_{2}$, the exit state of the second mission. The automaton $G$, corresponds to the possible movements of the AGV, it is an instance of the template $G_{T}$ with the initial state $q_{0}$ and the final state $q_{2}$. The supervisor must keep only all paths without blocking leading to the first 
state $q_{1}$, then at $q_{2}$. To formalize this problem we express this specification in the temporal logic called computation tree logic (CTL) (Clarke et al. 1986). The system must guarantee the CTL formula:

$$
\left(A F q_{1}\right) \text { and }\left(A G\left(q_{1} \Rightarrow A F q_{2}\right)\right) \text {. }
$$

In other words, $A F q_{1}$ means for all paths, we reach the state $q_{1}$ and $A G\left(q_{1} \Rightarrow A F q_{2}\right)$ is the classic response property that means starting from state $q_{1}$ system necessarily reaches the state $q_{2}$. The implementation of the supervisor must guarantee the previous formula. However, a theoretical maximally permissive model of a supervisor allows loops in the model and then, our supervisor must only guarantee the following property:

$$
\left(A G E F q_{1}\right) \text { and }\left(A G\left(q_{1} \Rightarrow A G E F q_{2}\right)\right)
$$

Since with the supervisory control theory we know effectively how to deal with a reachability problem, we decompose this complex problem into two simple reachability problems. The method used is to consider two separate missions that we sequentialize before computing the supervisor. The construction will ensure the reachability of the objective state of the first mission $\left(q_{1}\right)$ provided that the objective of the second mission $\left(q_{2}\right)$ is reached. The synthesis is as follows: we create two instances of the template $G_{T}=\left(Q_{T}, \Sigma_{T}, \delta_{T}, \emptyset, \emptyset\right)$ that we name $G^{1}$ and $G^{2}$. The initial state of $G^{1}$ is $q_{0}$ and its final state is $q_{1}$. The initial state of $G^{2}$ is $q_{1}$ and its final state is $q_{2}$. Thus, we have $G^{1}=\left(Q_{T}, \Sigma_{T}, \delta_{T}, q_{0},\left\{q_{1}\right\}\right)$ and $G^{2}=\left(Q_{T}, \Sigma_{T}, \delta_{T}, q_{1},\left\{q_{2}\right\}\right)$. We perform chaining by performing composition restricted on the set of states $Q_{\oplus}$ as follows:

$$
G=\left(Q_{\oplus}, \Sigma_{T}, \delta_{\oplus},\left(q_{0}, q_{1}\right),\left\{\left(q_{1}, q_{2}\right)\right\}\right)=G^{1} \oplus G^{2},
$$

where

$$
Q_{\oplus} \subseteq Q_{T} \times Q_{T}=\left\{\left(q, q^{\prime}\right) \in Q_{T} \times Q_{T} \text { such as } q=q_{1} \text { or } q^{\prime}=q_{1}\right\},
$$

and

$$
\delta_{\oplus}((x, y), \sigma)= \begin{cases}\left(x^{\prime}, y\right) & , \text { if } \delta_{T}(x, \sigma)=x^{\prime} \text { and }\left(x^{\prime}, y\right) \in Q_{\oplus} \\ \left(x, y^{\prime}\right) & , \text { if } \delta_{T}(y, \sigma)=y^{\prime} \text { and }\left(x, y^{\prime}\right) \in Q_{\oplus}\end{cases}
$$

Theorem 3 The supervisor $S\left(G^{1} \oplus G^{2}\right)$ ensures the properties $\left(A G E F\left(q_{1}, q_{1}\right)\right)$ and $\left(A G\left(\left(q_{1}, q_{1}\right) \Rightarrow A G E F\left(q_{1}, q_{2}\right)\right)\right)$ and it is maximally permissive.

Proof First, the state $\left(q_{2}, q_{1}\right)$, if it exists in $Q_{\oplus}$, is not final because only $\left(q_{1}, q_{2}\right)$ is the final state. The construction $G^{1} \oplus G^{2}$ ensures that if a path leads to the state $\left(q_{1}, q_{2}\right)$ then this path goes through the state $\left(q_{1}, q_{1}\right)$. The supervisor $S\left(G^{1} \oplus G^{2}\right)$ by definition ensures the property $A G E F\left(q_{1}, q_{2}\right)$ and therefore, by construction of $G^{1} \oplus G^{2}, A G E F\left(q_{1}, q_{1}\right)$ and $\left(A G\left(\left(q_{1}, q_{1}\right) \Rightarrow A G E F\left(q_{1}, q_{2}\right)\right)\right.$. Furthermore, any path in $G^{1}$ between $q_{0}$ and $q_{1}$ exists in $G^{1} \oplus G^{2}$ between $\left(q_{0}, q_{1}\right)$ and $\left(q_{1}, q_{1}\right)$, and any path in $G^{2}$ between $q_{1}$ and $q_{2}$ exists in $G^{1} \oplus G^{2}$ between $\left(q_{1}, q_{1}\right)$ and $\left(q_{1}, q_{2}\right)$. The computation of $S$ retains all safe paths and therefore the supervisor $S\left(G^{1} \oplus G^{2}\right)$ is maximally permissive. 
From this maximally permissive supervisor, a strategy to obtain an implementation satisfying the formula (3), consists classically in avoiding loop in the automaton. Another way we will study in further works to solve this problem consists in adding some criteria such as the energy consumption hence avoiding infinite loop.

\section{Experimental results}

The compositional approach presented in this paper has been implemented in $\mathrm{C}++$ and has been used on a standard $\mathrm{PC}$ with a single core $(3.2 \mathrm{GHz})$ and $8 \mathrm{~Gb}$ of memory (RAM). We studied four different real circuits from the industrial world and more precisely in the company BA Systèmes (France). However, the circuits 1 and 4 are the most significant because the configuration is often found in an industrial environment. The four configurations are different in terms of potential conflicts.

For the first circuit, we tested our approach on the area presented in Fig. 1. The circuit structure is very restrictive because interleaving is large whatever the system's state of progress. Indeed, looking at Fig. 3, we can access most states from any state of the automaton, which will cause a combinatorial explosion. For the second, the circuit respects the LIFO model. The first AGV to arrive will be the last to leave the area, therefore interleaving is limited. In this model, we have a lot of forbidden states and our method should be effective. For the third case, it is similar to the simple example presented in the section 3.6. In this configuration, there are few forbidden states and the compositional supervisor should be less effective than for the first two configurations. Finally, in the fourth configuration, the template authorizes to go into reverse everywhere whereas as we can see in Fig. 3 it is not possible. For example, if an AGV comes from $C M$ it cannot directly go on $E B$. In the industrial world, the interdiction to go into reverse is often a security requirement because a human who needs to go to the site must know in which direction the AGVs will move. Therefore this configuration is less realistic than the circuit 1 but the combinatory explosion is the biggest. However, this model contains 10 workstations so it is more realistic in terms of number of workstations.

We present in Table 2, the maximum memory requested and the time used to synthesize the supervisors for the previous cited cases. It is important to recall that our compositional approach provides exactly the same supervisor as the monolithic supervisor which is the most permissive.

1st circuit: In this example, we consider four AGVs and the fourth arrives when the first three are performing their missions, and a second case with a fifth AGV. All AGVs have two tasks, one going to the area and the second outside the area. $A G V_{1}$ (resp. $A G V_{2}, A G V_{3}, A G V_{4}$ ) starts on Enter ${ }_{1}$, (resp. Enter $_{2}$, Enter $_{3}$, Enter $_{4}$ ), has to go to the workstation $B_{1}$ (resp. $B_{4}, B_{2}, B_{3}$ ), 


\begin{tabular}{cccccccccc}
\hline \multicolumn{2}{c}{ Configuration } & & \multicolumn{3}{c}{ Monolithic } & & \multicolumn{3}{c}{ Compositional } \\
\cline { 1 - 2 } \cline { 8 - 10 } Circuit & AGVs & & States & Trans. & Time & & States & Trans. & Time \\
\hline 1 & 4 & & 332,856 & $1,443,504$ & $44 \mathrm{~s}$ & & 127,068 & 372,645 & $3.3 \mathrm{~s}$ \\
1 & 5 & & explosion & - & - & & $1,686,462$ & $2,529,639$ & $42 \mathrm{~s}$ \\
\hline 2 & 4 & & 782,130 & $4,000,492$ & $49 \mathrm{~s}$ & & 19,538 & 43,171 & $0.3 \mathrm{~s}$ \\
2 & 5 & & explosion & - & - & & 57,366 & 71,192 & $0.9 \mathrm{~s}$ \\
2 & 6 & & explosion & - & - & & 122,538 & 324,620 & $3.5 \mathrm{~s}$ \\
\hline 3 & 5 & & 27,720 & 120,031 & $1 \mathrm{~s}$ & & 24,920 & 107,341 & $0.4 \mathrm{~s}$ \\
3 & 6 & & 194,040 & $1,006,537$ & $10.1 \mathrm{~s}$ & & 174,440 & 900,907 & $3.4 \mathrm{~s}$ \\
\hline 4 & 3 & 15,372 & 47,854 & $1.1 \mathrm{~s}$ & & 12,646 & 36,182 & $0.2 \mathrm{~s}$ \\
4 & 4 & 937,692 & $4,148,854$ & $180 \mathrm{~s}$ & & 560,023 & $2,113,323$ & $19 \mathrm{~s}$ \\
\hline
\end{tabular}

Table 2 Experiments results. AGVs is the number of AGVs. The States, Trans. columns represent the maximum number of states and transitions generated during the synthesis and the time column is the time to synthesize the supervisor.

and leaves out by Exit (resp. Exit $_{2}, E_{\text {Exit }}$, Exit $_{4}$ ). For this example, we first consider three AGVs are performing their missions and a fourth arrives. $A G V_{1}$ completed its first mission and will leave the area $(E B), A G V_{2}$ starts its second mission $(K)$ and $A G V_{3}$ is performing its first task $(K I)$. The results are shown in Table 2. Next, we add a fifth AGV which arrives on Enter 2 and has to go to the workstation $B 1$. For this AGV we don't know its second mission. Note that the compositional approach is approximately thirteen times faster in this case, which provides an interesting time saver. This is explained by the reasons mentioned above, i.e., the reduction of the intermediate state space. These results confirm the explanation provided in section 3.3. Note that the compositional supervisor requires roughly four times less memory than the monolithic approach whereas for five AGVs the monolithic synthesis explodes.

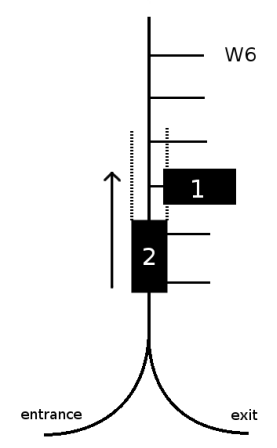

Fig. 9 An area which respects the LIFO model 
2nd circuit: The second case is also a realistic case, but it should be more favorable. Indeed, the automaton template has 30 states and 42 transitions but its main feature is to respect the LIFO model, there is only one exit from the area. This circuit is shown in Fig. 9. This type of configuration is often present in industrial site with limited space. If two or more AGVs go into the area, the first will be the last to leave because it will be blocked by the others (an AGV on the workstation blocks the central lane of the area). There is no solution except to wait for the other AGVs to have evacuated the area. For example, in Fig. 9, $A G V_{2}$ has to go to the workstation $W_{6}$ but it is impossible since $A G V_{1}$ blocks the central lane. $A G V_{2}$ has to wait in the entrance of the area until $A G V_{1}$ leave the area. We consider in this case four AGVs and the fourth arrives when the first three are performing their missions. $A G V_{1}$ achieved its first mission ( $0 \%$ of its second mission), $A G V_{2}$ performed $80 \%$ of its first mission and $A G V_{3} 70 \%$ of its first mission. The results are shown in Table 2. We also test for five and six AGVs with the following configuration: an AGV has completed its first mission, two are at $90 \%$ and two at $50 \%$ of their first mission, and the last AGV arrives at the entrance to the area.

With this circuit structure, the compositional approach is very efficient (almost 163 times faster). This is explained by the number of states that are forbidden caused by the LIFO model. Intermediate supervisors help to maintain a system with few states while the standard approach will calculate many forbidden states and will remove them very late. We note that for five or six AGVs, the monolithic approach explodes whereas our approach allows to generate the supervisor in $4.2 \mathrm{~s}$. We also note very significant gains in terms of memory space as we can see in the Table 2 .

3rd circuit: For the third example, the circuit used is similar to the simple example. It contains six workstations and we can have conflicts only on two places in the circuit (for example, the states $B$ and $D$ of the Fig. 6). Therefore the list of forbidden states is very small. The configuration is as follows: $A G V_{1}$ achieved its first mission (50\%), $A G V_{2}$ (resp. $A G V_{3}, A G V_{4}, A G V_{5}$ ) is achieving its first mission (45\%, resp. 20\%, 25\%, 25\%), and the $A G V_{6}$ arrives at the entrance of the area.

The model used is very small (46 states and 50 transitions) because each workstation has a unique path to reach it. Therefore an automaton $G_{i}$ has a maximum of 11 states and 10 transitions, that is why the monolithic approach can generate the supervisor for six AGVs in this case. As we can see in the Table 2, the space required for the monolithic approach is near to the space required for the compositional approach because the list of forbidden states is small. However, the compositional approach is once again faster than the monolithic one.

4th circuit: In the 4th case, three AGVs are moving on the area and a fourth arrives. The template automaton contains 58 states and 76 transitions. The configuration is as follows: $A G V_{1}$ is leaving the area (95\% of its mission has performed), $A G V_{2}$ achieved its first mission (50\%, it is in a workstation), $A G V_{3}$ 
is performing its first mission (15\%) and the $A G V_{4}$ arrives at the entrance of the area.

As explained previously, this circuit causes a large combinatorial explosion and this is mainly why the monolithic approach is very slow. As we can see in the Table 2, the standard approach has twice more transitions and states than the compositional approach. Therefore the compositional approach is faster than the standard approach.

\section{Conclusion}

In this paper, we propose a compositional approach (modified monolithic supervisor) to synthesize an on-line supervisor to manage a fleet of mobile robots. We prove compositionality of supervisor so that the supervisor is the same as the monolithic approach of Ramadge and Wonham. This method has several advantages. First, it is more efficient than the standard approach, and according to the case it allows to synthesize a supervisor for four or more AGVs in a critical area, which is not possible with the monolithic approach. But the online application in a real time context is the biggest advantage for this method as explained in previous sections. Indeed, in an industrial environment, a mission is often generated in real time, we cannot know it in advance. Therefore, it is essential to be able to generate a supervisor when a new task appears in its critical areas.

This paper focuses only on critical areas, if we extend to the non-critical areas (lack of interaction), we could use local controllers. This will be the subject of a future study.

In our future work, we would like to add a notion of weight on transitions to choose the best path in real time according to some criteria, such as energy or time and we will implement this approach in company BA Systèmes (France).

\section{References}

Arnaud Y, Cury JER, Loiseau JJ, Martinez C (2009) Pilotage sûr et optimal d'une flotte de véhicules autoguidés. In: JD-JN-MACS 2009, Angers : France

Breton L, Maza S, Castagna P (2006) A multi-agent based conflict-free routing approach of bi-directional automated guided vehicles. In: American Control Conference, 2006

Cai K, Wonham WM (2010) Supervisor localization: A top-down approach to distributed control of discrete-event systems. Automatic Control, IEEE Transactions on 55(3):605-618, DOI 10.1109/TAC.2009.2039237

Cassandras CG, Lafortune S (2006) Introduction to Discrete Event Systems. Springer-Verlag New York, Inc., Secaucus, NJ, USA 
Clarke EM, Emerson EA, Sistla AP (1986) Automatic verification of finitestate concurrent systems using temporal logic specifications. ACM Trans Program Lang Syst 8(2):244-263, DOI 10.1145/5397.5399

Flordal H, Malik R, Fabian M, Åkesson K (2007) Compositional synthesis of maximally permissive supervisors using supervision equivalence. Discrete Event Dynamic Systems 17(4):475-504, DOI 10.1007/s10626-007-0018-z

Girault J, Loiseau JJ, Roux OH (2013) Synthèse en ligne de superviseur compositionnel pour flotte de robots mobiles. In: European Journal of Automation, MSR'13, vol 47/1-3, pp 195-210

Hill RC, Tilbury DM (2006) Modular supervisory control of discrete-event systems with abstraction and incremental hierarchical construction. In: Discrete Event Systems, 2006 8th International Workshop on, pp 399-406, DOI 10.1109/WODES.2006.382507

Komenda J, Van Schuppen J, Gaudin B, Marchand H (2008) Supervisory control of modular systems with global specification languages. Automatica 44:1127-1134, DOI 10.1016/j.automatica.2007.09.004

Krogh BH, Holloway LE (1991) Synthesis of feedback control logic for discrete manufacturing systems. Automatica 27(4):641-651

Malik R, Flordal H (2008) Yet another approach to compositional synthesis of discrete event systems. In: Discrete Event Systems, 2008. WODES 2008. 9th International Workshop on, pp 16-21, DOI 10.1109/WODES.2008.4605916

Maza S (2003) Analyse du comportement d'un système de transport par chariot bidirectionnels en vue de sa commande. $\mathrm{PhD}$ thesis, Université de Nantes

Mohajerani S, Malik R, Ware S, Fabian M (2011) Compositional synthesis of discrete event systems using synthesis abstraction. In: Control and Decision Conference (CCDC), 2011 Chinese, pp 1549-1554, DOI 10.1109/CCDC.2011.5968439

Queiroz MHD, Cury JER (2000) Modular supervisory control of large scale discrete event systems. In: International Workshop on Discrete Event Systems: Analysis and Control. Proc. WODES'00, Kluwer Academic, pp 103-110

Ramadge PJ, Wonham WM (1987) Supervisory control of a class of discrete event processes. SIAM J Control Optim 25(1):206-230

Ramadge PJ, Wonham WM (1989) The control of discrete event systems. Proceedings of the IEEE 77(1):81-98

Reveliotis SA (2000) Conflict resolution in agv systems. IIE Transactions $32: 200-0$

Wonham WM, Ramadge PJ (1984) On the supremal controllable sublanguage of a given language. In: Decision and Control, 1984. The 23rd IEEE Conference on, vol 23, pp 1073-1080

Wonham WM, Ramadge PJ (1988) Modular supervisory control of discreteevent systems. Mathematics of Control, Signals and Systems 1(1):13-30 